\title{
Cardiovascular Safety of Nilotinib in Alzheimer's Disease
}

Sean Tan MBBS $S^{1}$, Diva Baggio $M B B S^{2}$, Jake Shortt BMedSc MBChB PhD ${ }^{2,3}$, Brian Ko BSc

MBBS MD, $P h D^{1,4}$

${ }^{1}$ Monash Heart, Monash Health, Clayton, Victoria, Australia

${ }^{2}$ Monash Haematology, Monash Health, Clayton, Victoria, Australia

${ }^{3}$ School of Clinical Sciences at Monash Health, Faculty of Medicine Nursing and Health Sciences, Monash University, Clayton, Victoria

${ }^{4}$ Monash Cardiovascular Research Centre, Monash University, Clayton, Victoria, Australia

\section{Running Head:}

Cardiac Safety of Nilotinib in Alzheimer's Disease

\section{Address for Correspondence:}

A/Prof Brian Ko

MonashHeart, Monash Health, 246 Clayton Road, Clayton, Victoria 3168, Australia

Email : brianshiuhangko@ gmail.com

Tel: $\quad$ +61395946666

Fax: $\quad$ +61395946239 
Number of Characters in Title: 57

Number of Characters in Running Head: 50

Word count: 400

Number of Figures: 0

Number of Tables: 0 


\section{Manuscript}

We read with interest the article by Turner et al on the use of Nilotinib for the treatment of mild-to-moderate Alzheimer's disease $(\mathrm{AD})^{1}$. In this phase 2 trial, Nilotinib was found to reduce central nervous system amyloid burden, cerebrospinal fluid $\mathrm{A} \beta 40$ and $\mathrm{A} \beta 42$, hippocampal volume loss and phospho-tau-181 associated with $\mathrm{AD}$ at 12 months follow-up ${ }^{1}$. The authors reported the cardiac safety profile of Nilotinib, and demonstrated no QTc prolongation which can be associated with risk of sudden cardiac death ${ }^{1}$.

We caution that the risk and incidence of cardiovascular events (CVE) while on Nilotinib may not have been fully accounted in this report. In the ENESTnd trial which investigated the efficacy and safety of Nilotinib in the treatment of chronic myeloid leukaemia, there was a doseand duration-dependent association between Nilotinib and CVE, defined as a composite of ischaemic heart disease, ischaemic cerebrovascular events and peripheral vascular disease ${ }^{2,3}$. This trial initially described no CVE at 12 months $^{2}$, yet the 5-year CVE incidence was $7.5 \%$ in patients receiving Nilotinib 300mg twice daily and $13.4 \%$ with $400 \mathrm{mg}$ twice daily ${ }^{3}$. Patients' baseline Framingham cardiovascular risk score was found to be predictive for $\mathrm{CVE}^{3}$. Furthermore, Nilotinib was found to significantly increase low-density-lipoprotein cholesterol and glycated haemoglobin to above clinical thresholds ${ }^{3}$.

Patients receiving Nilotinib in the study by Turner et. al. had a mean age of 72 years and were predominantly female. Baseline cardiovascular risk factors, scores and prevalence of known CVE prior to trial entry were not reported. Based on mean age of participants, it may be reasonable to assume a significant proportion of trial population may have moderate-to-high cardiovascular risk. 
While the daily dose and duration of Nilotinib used in this trial (150mg for 6 months followed by $300 \mathrm{mg}$ for 6 months) were lower than that in ENESTnd trial, cardiovascular risk upon prolonged use in moderate/high cardiovascular risk patients remains unknown and should be considered in future trials. Finally, the authors suggested that these doses may be non-cardiotoxic based on previously reported lack of plasma Abelson inhibition using similarly low doses of Nilotinib $^{1}$. It is worth noting that Nilotinib toxicity may result from both on-target and offtarget kinase inhibition, hence may not be predicted by plasma Abelson inhibition alone ${ }^{4}$.

In summary, low dose Nilotinib may be a promising treatment for $\mathrm{AD}$, however assessment of cardiovascular risk and detailed description of acute and long term CVE should be considered in the future phase 3 trials.

\section{Potential Conflicts of Interest}

J.S. has received honoraria and served on advisory committee for Novartis, which manufactures Nilotinib that is discussed in this letter. J.S. has also received honoraria from Bristol Myer Squibb, which manufactures the tyrosine kinase inhibitor Dasatinib, and Specialised Therapeutics Australia, which manufactures the tyrosine kinase inhibitor Ponatinib.

B.K. has received honoraria and served on advisory committee for Novartis, which manufactures Nilotinib that is discussed in this letter. B.K. has also received honoraria from Bristol Myer Squibb, which manufactures the tyrosine kinase inhibitor Dasatinib, and Specialised Therapeutics Australia, which manufactures the tyrosine kinase inhibitor Ponatinib.

Monash Haematology has received research funding from Bristol Myer Squibb, which manufactures the tyrosine kinase inhibitor Dasatinib.

The remaining authors have nothing to disclose. 


\section{References}

1. Turner RS, Hebron ML, Lawler A, et. al. Nilotinib effects on safety, tolerability, and biomarkers in Alzheimer's disease. Ann Neurol. 2020;88(1):183-194.

2. Saglio G, Kim D-W, Issaragrisil S, et. al. Nilotinib versus imatinib for newly diagnosed chronic myeloid leukaemia. N Engl J Med. 2010;362(24):2251-2259.

3. Hochhaus A, Saglio G, Hughes TP, et. al. Long-term benefits and risks of frontline nilotinib vs imatinib for chronic myeloid leukaemia in chronic phase: 5-year update of the randomized ENESTnd trial. Leukemia. 2016;30(5):1044-1054.

4. Kim TD, le Coutre P, Schwarz M, et. al. Clinical cardiac safety profile of nilotinib. Haematologica. 2012;97(6):883-889. 


\section{University Library}

\section{- M M N E R VA A gateway to Melbourne's research publications}

Minerva Access is the Institutional Repository of The University of Melbourne

Author/s:

Tan, S;Baggio, D;Shortt, J;Ko, B

Title:

Cardiovascular Safety of Nilotinib in Alzheimer Disease.

Date:

2021-01

Citation:

Tan, S., Baggio, D., Shortt, J. \& Ko, B. (2021). Cardiovascular Safety of Nilotinib in Alzheimer Disease.. Ann Neurol, 89 (1), pp.196-. https://doi.org/10.1002/ana.25947.

Persistent Link:

http://hdl.handle.net/11343/276583 